\title{
Healthcare, Medication Utilization and Outcomes of Patients with COPD by GOLD Classification in England
}

\author{
Leah B Sansbury' \\ Kieran J Rothnie ${ }^{2}$ \\ Chanchal Bains ${ }^{3}$ \\ Chris Compton ${ }^{4}$ \\ Glenn Anley ${ }^{2}$ \\ Afisi S Ismaila $\mathbb{D}^{5,6}$
}

'Value Evidence and Outcomes, GlaxoSmithKline, Research Triangle Park, Durham, NC, USA; ${ }^{2}$ Value and Evidence Outcomes, GlaxoSmithKline, Uxbridge, UK; ${ }^{3}$ Real World Evidence and Epidemiology, GlaxoSmithKline, Uxbridge, UK; ${ }^{4}$ Global Respiratory Franchise, GlaxoSmithKline, Brentford, UK; ${ }^{5}$ Value Evidence and Outcomes, GlaxoSmithKline, Collegeville, PA, USA; ${ }^{6}$ Department of Health Research Methods, McMaster University, Hamilton, ON, Canada
Correspondence: Afisi S Ismaila Value Evidence and Outcomes, GlaxoSmithKline, 1250 South Collegeville Road, Collegeville, PA, 19426-0989, USA Tel + I 9193158229

Email afisi.s.ismaila@gsk.com
Background: Available data on the relationship between COPD symptoms, disease outcomes, and mortality are currently limited. This study investigated the clinical characteristics, outcomes, healthcare utilization, and prescribing practices across GOLD 2017 groups (A, B, C, and D) in a large-scale, population-based cohort of COPD patients managed in an English primary care setting.

Patients and Methods: This retrospective analysis included patients aged $\geq 35$ years, with a confirmed diagnosis of COPD and $\geq 1$ record of pulmonary function testing in their medical history. Medical Research Council dyspnea score and exacerbation history were used to define patients' GOLD 2017 classification. Patients were identified using the UK Clinical Practice Research Database and were followed for 12 months.

Results: Eligible COPD patients' ( $\mathrm{N}=42,331$; mean [SD] age, 69.5 [10.7] years; 54\% males), GOLD 2017 categorizations were: Group A: 49.1\%, Group B: 30.5\%, Group C: $8.2 \%$, Group D: $12.1 \%$. Overall, $37.7 \%$ of patients experienced $\geq 1$ moderate COPD exacerbation. The rate of moderate exacerbations per person per year (PPPY) was highest in GOLD group D (0.72), followed by C (0.53), B (0.22), and A (0.15), while the rate of exacerbations leading to hospitalization PPPY was much higher in D $(0.27)$ than in B $(0.10), C(0.08)$, or A (0.03). Overall, 56.4\% of patients visited their general practitioner $\geq 5$ times in the 12 months of follow-up. Time-to-event analysis suggested that breathlessness contributed to exacerbation severity and frequency. One-year mortality was highest in GOLD groups D and B. The most frequent prescribed maintenance therapies were inhaled corticosteroids with long-acting $\beta_{2}$-agonists, multiple-inhaler triple therapy, or long-acting muscarinic antagonist, irrespective of GOLD classification.

Conclusion: The burden of COPD remains substantial in England. Stratification of this large primary care population according to GOLD criteria predicted the risk of COPD exacerbations. Understanding populations of patients with COPD may enable the optimization of patient care.

Keywords: chronic obstructive lung disease, database, exacerbations, healthcare costs, Global Initiative for Chronic Obstructive Lung Disease, maintenance therapy

\section{Introduction}

Chronic obstructive pulmonary disease (COPD) is a major cause of morbidity and mortality that presents a significant burden on healthcare systems and resources worldwide, ${ }^{1}$ including healthcare costs. ${ }^{2}$ Indeed, the World Health Organization lists COPD as the third most common cause of death worldwide. ${ }^{3}$ The Global Initiative for Chronic Obstructive Lung Disease (GOLD) guidelines provide a classification system 
to assess disease severity among patients with COPD, in which patients are assigned to one of four GOLD classification groups (A, B, C, or D) that allow suitable maintenance treatment options to be recommended. ${ }^{4,5}$ In updated guidelines published in 2017, a simplified tool for assessing COPD severity based on exacerbation history and respiratory symptoms was defined..$^{5}$ By moving away from grading exacerbation risk based on airflow limitation and exacerbation history, to exacerbation history alone, the distribution of patients across the GOLD 2017 groups has been altered compared with the GOLD 2013 system; mostly by the reclassification of patients from group $\mathrm{C}$ to group $\mathrm{A}$, and from group $\mathrm{D}$ to group B. ${ }^{6-8}$

Recent studies in COPD have focused on the prognostic value of acute exacerbations on the risk of future exacerbations and mortality. ${ }^{9-14}$ However, relatively less attention has been paid to the relationship between COPD symptoms and disease outcomes, and death, despite some studies indicating an association between dyspnea severity and the risk of exacerbations and hospitalizations. ${ }^{15-19}$ Further research is, therefore, needed in this area in order to elucidate the effect of these clinical characteristics.

Studies have confirmed that Clinical Practice Research Datalink (CPRD) data are of sufficient completeness and quality to assess digital, COPD-related data recorded by general practitioners (GPs) in the UK..$^{20,21}$ In order to ensure that the best patient care is provided, and that healthcare resource utilization is minimized where possible, it is important to assess how closely real-world prescribing practices reflect published recommendations for COPD management. $^{22}$ Here, we have used data from two linked databases (CRPD and Hospital Episode Statistics [HES]) to investigate clinical characteristics, outcomes, healthcare utilization, and prescribing practices across GOLD 2017 groups (A, B, C, and D) in a large-scale, population-based cohort of COPD patients managed in an English primary care setting. As part of this analysis, we investigated the 1-year risk of exacerbations and mortality among GOLD group classification and among patients with different degrees of breathlessness (graded using the Medical Research Council [MRC] dyspnea scale).

\section{Materials and Methods}

\section{Study Design}

The design of this retrospective database cohort study is shown in Figure 1.

\section{CPRD and HES}

Data on COPD patients treated in primary care in England were extracted from the CPRD database, which contains anonymized longitudinal medical records from primary care across England (and the rest of the UK). In the CPRD database, data containing all records deemed relevant to patient care by the GP are drawn from computer systems used within general practice to maintain clinical records. GOLD 2017 data were derived based on availability of longitudinal individual patient records containing information on moderate (GP-treated) COPD exacerbations, airflow

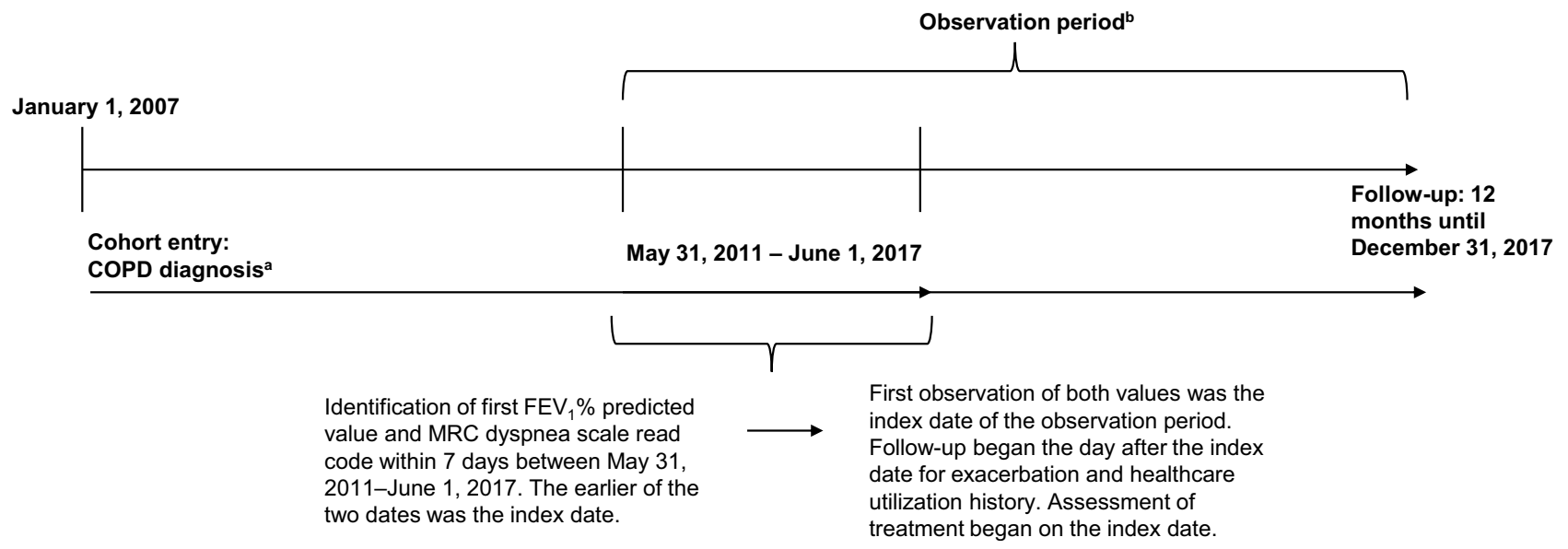

Figure I Study design. ${ }^{a}$ Confirmed by spirometry, FEV $/ F V C<0.7$ (any time). ${ }^{b}$ Future outcomes ascertained from the database from the defined index date until 90 days post index date for treatment and the day after the defined index date and 12 months post index date, until December 3I, 20I7, for categorical exacerbations per year and health outcomes of interest.

Note: Healthcare utilization and longitudinal outcomes (GP visits, hospitalizations, and COPD exacerbations per year) were analyzed in patients who had at least 12 months of follow-up data after the index date in the CPRD database.

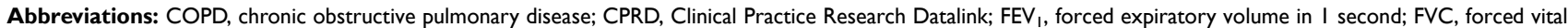
capacity; GP, general practitioner; MRC, Medical Research Council. 
limitation, and MRC dyspnea scale as a measure of COPD symptoms. The extracted anonymized patient data were individually linked to secondary care data in the HES database, which contains records of inpatient and outpatient care, and attendance at Accident and Emergency National Health Service (NHS) hospitals in England. HES data were used to ascertain severe (hospitalized) COPD exacerbations. CPRD linkage to HES data has been used as a method for assessing healthcare utilization and informing provision of healthcare services in England. ${ }^{23}$ CPRD-HES linkage in this study was completed by NHS Digital (Leeds, UK).

\section{Inclusion and Exclusion Criteria}

To be included in the cohort, patients $\geq 35$ years of age were required to have one or more records for COPD diagnosis in the CPRD database between January 1, 2007 and June 1, 2017, including at least one record of a forced expiratory volume in 1 second/forced vital capacity $\left(\mathrm{FEV}_{1}\right.$ (FVC) ratio of $<0.7$ at any time in their medical history. After cohort entry, patients were required to have at least one $\mathrm{FEV}_{1} \%$ predicted value and MRC dyspnea scale read code recorded within 7 days between May 31, 2011 and December 31, 2017. The first observation of both values (within 7 days) was defined as the index date.

Patients were required to have a database history of $\geq 12$ months available before, and including, the index date, and 90 days after the index date, up until December 31, 2017. They were also required to have available data for CPRD-HES database linkage. Healthcare utilization and longitudinal outcomes (GP visits, hospitalizations, and COPD exacerbations per year) were analyzed in patients who had at least 12 months of follow-up data after the index date in the CPRD database. Patients were excluded if they had an occurrence of a code for a medical condition incompatible with COPD diagnosis at any time in their history, including conditions related to lung or bronchial developmental anomalies, degenerative processes, pulmonary resection or other significant respiratory disorders, or lung malformations other than COPD.

This study complied with all applicable laws regarding patient privacy. No direct patient contact or collection of additional patient data occurred. CPRD GOLD and linked CPRD-HES data contain only fully de-identified patient data, and no confidential information or patient identifiers were available to the study team. All data held and processed by CPRD were stored on a secure computer network and were compliant with the relevant legal obligations, including the Data Protection Act 1998. The study protocol was approved by the Independent Scientific Advisory Committee (ISAC) overseeing CPRD (ISAC 17 270).

\section{COPD Treatments}

Information on any treatment prescribed during the 90 days on or after the index date was collected. The following treatment groups were considered: short- and long-acting bronchodilators (short-acting muscarinic antagonists [SAMAs] and $\beta_{2}$-agonists [SABAs], and long-acting muscarinic antagonists [LAMAs] and $\beta_{2}$-agonists [LABAs]); inhaled corticosteroids (ICS); and theophylline. ICS, LAMA, and LABA could be prescribed individually, together in open combination (dual and triple therapies), or included as a fixed-dose dual-therapy combination in the case of ICS+LABA or LAMA+LABA (but not ICS+LAMA).

\section{Stratification According to GOLD Group and MRC Dyspnea Scale}

The patient population was categorized into GOLD 2017 groups $\mathrm{A}-\mathrm{D}$ and according to their MRC dyspnea grade. MRC dyspnea scores were used in this analysis instead of modified MRC scores, as regular recording of MRC scores is part of the Quality and Outcomes Framework for the care of COPD patients in the UK, as defined by National Institute for Health and Care Excellence guidelines, ${ }^{24}$ and as such, are more commonly recorded in the CPRD.

\section{Descriptive Analyses and Outcome Definitions}

Descriptive analyses were conducted in the overall patient population and in patients stratified according to COPD severity as defined by GOLD 2017. Objectives of these analyses were to (1) evaluate the distribution of patients with COPD by GOLD group, along with the distribution of each component of GOLD classification within each group (specifically spirometry classification, number of exacerbations per year, and MRC dyspnea grade); (2) evaluate the demographics, clinical characteristics, healthcare utilization (primary care [GP] consultation visits and hospitalizations), and disease burden (MRC dyspnea grade and number of exacerbations) in patients with COPD in the 12 months prior to the index date; (3) describe the treatment(s) received by patients with COPD during the 90 days after, and including, the index date; and (4) describe the number of moderate (treated in primary care) and/or 
severe exacerbations (requiring hospitalization), and mortality in patients with COPD 12 months after the index date. For these descriptive analyses, categorical variables were summarized as number of patients (or events) and percentages. Continuous variables were presented as mean (standard deviation [SD]). COPD exacerbations in the 12 months prior to, and including, the index date were described by number of patients and percentage. Moderate and severe COPD exacerbations were categorized into 0,1 , and $\geq 2$ prior events per patient, with overall number of patients and percentage presented for each category. To define the rate of exacerbations per patient per year (PPPY), patients without 12 months of follow-up information were censored at the time of cohort exit, unenrollment, death, or at the end of follow-up on December 31, 2017. In the healthcare utilization assessment, patients were classed as having between 0 and $\geq 5$ GP visits in the 12 months prior to, and including, the index date.

\section{Time-to-Event Analyses for COPD Exacerbations and Death in Patients Stratified According to GOLD 2017 and MRC Dyspnea Grade}

Univariable Cox proportional hazards models investigated the time to first moderate (primary care treated) or severe (hospitalized) COPD exacerbation, and time to death in the overall study population, stratified according to GOLD 2017 or MRC dyspnea grade. In the GOLD 2017 analysis, the hazard was modeled as a function of GOLD group, with GOLD group A set as the reference group for risk calculations. For the MRC dyspnea analysis, the hazard was modeled as a function of dyspnea grade, with grade 1 set as the reference group, such that the hazard ratio (HR), 95\% confidence intervals (CIs) for HR, and log-rank $\mathrm{P}$ values comparing GOLD groups $\mathrm{B}, \mathrm{C}$, or $\mathrm{D}$ versus GOLD group A, or MRC dyspnea grade 2, 3, 4, or 5 versus grade 1 , are presented. The cumulative proportion of patients with events was calculated and plotted per GOLD group or MRC dyspnea grade, with patients being followed up for COPD exacerbations for 12 months following index date. Patients were followed up for death from 90 days post index until 12 months post index (patients were required to survive at least 90 days after the index date to be eligible for the study). For the analyses of time to COPD exacerbation (moderate or severe) and death, patients were censored at time of cohort exit, unenrollment, death, or the end of follow-up (June 1, 2017).

\section{Results}

\section{Patient Baseline Demographics and Clinical Characteristics Prior to Index Date}

A total of 42,331 patients (mean age 70 years, $54.4 \%$ male) were included in the study, and classified into GOLD 2017 groups. Most patients were current (39.3\%) or former (54.8\%) smokers, and $40.0 \%$ had MRC grade 2 dyspnea. In total, $14.1 \%$ of patients had a current asthma diagnosis. Patient demographics and clinical characteristics, overall and by GOLD 2017 group, are presented in Table 1. Most patients were classified as GOLD group A $(n=20,788)$, while GOLD group $C$ contained the fewest patients $(\mathrm{n}=3485)$.

In the total cohort, $58.8 \%$ of patients visited their GP $\geq 5$ times, while $5.4 \%$ did not visit their GP at all in the 12 months prior to, and including, the index date (Table 1). These proportions were similar among patients who had not received maintenance therapy: $53.1 \%$ of these patients visited their GP $\geq 5$ times and $6.4 \%$ did not visit their GP at all. The proportion of patients who had visited their GP $\geq 5$ times in the 12 months preceding the index date was highest in GOLD group D (78.5\%) (Table 1). Overall, $39.2 \%$ of patients had experienced $\geq 1$ moderate COPD exacerbation, and $4.5 \%$ of patients had experienced $\geq 1$ severe COPD exacerbation leading to hospitalization in the previous year (Table 1). Overall, 9502 patients ( $22.4 \%$ of the total population) had not received any longacting maintenance therapy in the 12 months prior to the index date. Among patients who had not received maintenance therapy prior to the index date, $25.4 \%$ had experienced $\geq 1$ moderate COPD exacerbation, and $1.7 \%$ had experienced $\geq 1$ severe COPD exacerbation in the year prior to the index date.

\section{COPD Exacerbations and Healthcare Utilization in the Year Following the Index Date}

Longitudinal outcomes and healthcare utilization in the year following the index date were generally similar across GOLD 2017 groups A-D (Table 2). In the overall cohort, $37.7 \%$ of patients experienced $\geq 1$ moderate COPD exacerbation, and $6.4 \%$ experienced $\geq 1$ severe COPD exacerbation 
Table I Patient Demographics and Clinical Characteristics, Overall and Stratified by GOLD 2017 Group

\begin{tabular}{|c|c|c|c|c|c|}
\hline & Overall & $\begin{array}{c}\text { GOLD } \\
\text { Group A }\end{array}$ & $\begin{array}{l}\text { GOLD } \\
\text { Group B }\end{array}$ & $\begin{array}{l}\text { GOLD } \\
\text { Group C }\end{array}$ & $\begin{array}{l}\text { GOLD } \\
\text { Group D }\end{array}$ \\
\hline Total, $\mathbf{n}$ (\% overall population) & $42,331(100)$ & $20,788(49.1)$ & $12,925(30.5)$ & $3485(8.2)$ & $5133(12.1)$ \\
\hline Age, mean (SD) years & $70(11)$ & $68(11)$ & $71(11)$ & $68(10)$ & $71(11)$ \\
\hline Male, $n$ (\%) & $23,037(54.42)$ & II,797 (56.75) & $6959(53.84)$ & 1763 (50.59) & $2518(49.06)$ \\
\hline \multicolumn{6}{|l|}{ Smoking status, n (\%) } \\
\hline Ex-smoker & $23,167(54.76)$ & II,009 (52.98) & $7274(56.32)$ & $1872(53.75)$ & $3012(58.68)$ \\
\hline Current smoker & $16,633(39.31)$ & 8351 (40.19) & $696(5.39)$ & |42| (40.77) & $1916(37.33)$ \\
\hline Never-smoker & $2509(5.93)$ & $1418(6.82)$ & 4945 (38.29) & $190(5.46)$ & 205 (3.99) \\
\hline FEV , at baseline (\% predicted) & $59.18(14.04)$ & $60.58(12.55)$ & $58.06(14.78)$ & $59.85(14.42)$ & $55.86(16.57)$ \\
\hline \multicolumn{6}{|l|}{ MRC dyspnea grade ${ }^{a}, n$ (\%) } \\
\hline Grade I & $7363(17.39)$ & $6581(31.66)$ & 0 & 782 (22.44) & 0 \\
\hline Grade 2 & $16,910(39.95)$ & $\mid 4,207(68.34)$ & 0 & $2703(77.56)$ & 0 \\
\hline Grade 3 & $10,985(25.95)$ & 0 & $8325(64.41)$ & 0 & $2660(51.82)$ \\
\hline Grade 4 & $6033(14.25)$ & 0 & $4019(31.09)$ & 0 & $2014(39.24)$ \\
\hline Grade 5 & $1040(2.46)$ & 0 & $581(4.50)$ & 0 & $459(8.94)$ \\
\hline \multicolumn{6}{|l|}{ Moderate COPD exacerbations ${ }^{b}, n(\%)$} \\
\hline 0 events & $25,728(60.78)$ & I5,463 (74.38) & 9059 (70.09) & $454(13.03)$ & $752(14.65)$ \\
\hline I event & $9822(23.20)$ & $5325(25.62)$ & $3866(29.91)$ & $187(5.37)$ & $444(8.65)$ \\
\hline$\geq 2$ events & $678 \mid(16.02)$ & 0 & 0 & $2844(8 I .6 I)$ & $3937(76.70)$ \\
\hline \multicolumn{6}{|l|}{$\begin{array}{l}\text { Hospitalized COPD exacerbations }{ }^{\mathrm{b}} \text {, } \\
\text { n (\%) }\end{array}$} \\
\hline 0 events & 40,421 (95.49) & $20,788(100)$ & $|2,92|(99.97)$ & $2902(83.27)$ & $3810(74.23)$ \\
\hline I event & $1644(3.88)$ & 0 & $4(0.03)$ & $548(15.72)$ & $1092(21.27)$ \\
\hline$\geq 2$ events & $266(0.63)$ & 0 & 0 & $35(1.00)$ & $231(4.50)$ \\
\hline \multicolumn{6}{|l|}{ GP practice visits ${ }^{b}, n(\%)$} \\
\hline 0 & $2269(5.36)$ & 1405 (6.76) & $676(5.23)$ & $75(2.15)$ & $113(2.20)$ \\
\hline $\mathbf{I}$ & $3248(7.67)$ & $2005(9.64)$ & 961 (7.44) & $218(3.67)$ & $154(3.00)$ \\
\hline 2 & $3919(9.26)$ & $2344(11.28)$ & $1163(9.00)$ & $186(5.62)$ & $216(4.21)$ \\
\hline 3 & $4107(9.70)$ & $2285(10.99)$ & $1260(9.75)$ & $265(7.60)$ & $297(5.79)$ \\
\hline 4 & $3904(9.22)$ & $2084(10.03)$ & $1207(9.34)$ & $290(8.32)$ & $323(6.29)$ \\
\hline$\geq \mathbf{5}$ & $24,884(58.78)$ & $10,665(51.30)$ & $7658(59.25)$ & $2531(72.63)$ & $4030(78.5 I)$ \\
\hline Mean per year (SD) & $7.0(6.1)$ & $5.9(5.2)$ & $7.0(6.0)$ & $8.6(6.1)$ & $10.4(7.7)$ \\
\hline
\end{tabular}

Notes: ${ }^{a}$ As recorded on the index date; In the 12 months prior to and including the index date.

Abbreviations: COPD, chronic obstructive pulmonary disease; FEV GP, general practitioner; MRC, Medical Research Council; SD, standard deviation.

leading to hospitalization in the year following the index date. Among the GOLD 2017 groups, the rate of moderate exacerbations PPPY was highest in GOLD group D (0.72), followed by groups C (0.53), B (0.22), and A (0.15), respectively. The rate of exacerbations leading to hospitalization PPPY was much higher in GOLD group $D(0.27)$ than in groups B (0.10), C (0.08), or A (0.03) (Table 2). In the total cohort, $56.4 \%$ of patients visited their GP at least five times in the 12 months following the index date, whereas only $6.8 \%$ did not visit their GP at all (Table 2). The rate of GP visits PPPY was higher in more symptomatic patients (9.64 in
GOLD group D and 7.22 in GOLD group B), than in less symptomatic patients (7.63 in GOLD group $\mathrm{C}$ and 5.84 in GOLD group A).

\section{Risk of COPD Exacerbations and Death in Patients with COPD According to GOLD 2017 Group}

The time to COPD exacerbation, and time to death in patients stratified according to the GOLD 2017 classification, is shown in Figure $2 \mathrm{~A}-\mathrm{C}$. Univariate regression 
Table 2 Longitudinal Outcomes and Healthcare Utilization of Patients in the 12 Months After the Index Date, Overall and Stratified by GOLD 2017 Group

\begin{tabular}{|c|c|c|c|c|c|}
\hline & Overall & $\begin{array}{l}\text { GOLD } \\
\text { Group A }\end{array}$ & $\begin{array}{l}\text { GOLD } \\
\text { Group B }\end{array}$ & $\begin{array}{l}\text { GOLD } \\
\text { Group C }\end{array}$ & $\begin{array}{l}\text { GOLD } \\
\text { Group D }\end{array}$ \\
\hline Total, n (\%) & $42,331(100)$ & $20,788(49.11)$ & $12,925(30.5)$ & $3485(8.23)$ & $5133(12.13)$ \\
\hline \multicolumn{6}{|c|}{ Moderate COPD exacerbations, n (\%) } \\
\hline 0 events & $26,375(62.31)$ & $15,118(72.72)$ & $8328(64.43)$ & II3I (37.68) & $1616(31.48)$ \\
\hline I event & $9118(21.54)$ & $3917(18.84)$ & $2964(22.93)$ & $908(26.05)$ & $1329(25.89)$ \\
\hline$\geq 2$ events & $6838(16.15)$ & $1753(8.43)$ & $1633(12.63)$ & $1264(36.27)$ & $2188(42.63)$ \\
\hline Rate PPPY $(95 \% \mathrm{Cl})$ & $0.27(0.27,0.28)$ & $0.15(0.14,0.15)$ & $0.22(0.22,0.23)$ & $0.53(0.5 \mathrm{I}, 0.55)$ & $0.72(0.69,0.74)$ \\
\hline \multicolumn{6}{|c|}{$\begin{array}{l}\text { Hospitalized COPD exacerbations, } \\
\text { n (\%) }\end{array}$} \\
\hline 0 events & $36,912(93.58)$ & $20,243(97.38)$ & II,892 (92.01) & $3257(93.46)$ & $4220(82.81)$ \\
\hline I event & $2136(5.05)$ & $488(2.35)$ & $844(6.53)$ & $195(5.60)$ & $609(11.86)$ \\
\hline$\geq 2$ events & $583(1.38)$ & $57(0.27)$ & $189(1.46)$ & $33(0.95)$ & $304(5.92)$ \\
\hline Rate PPPY $(95 \% \mathrm{Cl})$ & $0.08(0.08,0.09)$ & $0.03(0.03,0.03)$ & $0.10(0.09,0.11)$ & $0.08(0.07,0.09)$ & $0.27(0.26,0.29)$ \\
\hline \multicolumn{6}{|l|}{ GP practice visits, n (\%) } \\
\hline 0 & $2858(6.75)$ & $1758(8.46)$ & $760(5.88)$ & $167(4.79)$ & $173(3.37)$ \\
\hline I & $3694(8.73)$ & $2221(10.68)$ & $980(7.58)$ & $233(6.69)$ & $260(5.07)$ \\
\hline 2 & $4164(9.84)$ & $2372(\mid I .4 I)$ & $1166(9.02)$ & $301(8.64)$ & $325(6.33)$ \\
\hline 3 & $3940(9.31)$ & $2180(10.49)$ & II $144(8.85)$ & $306(8.78)$ & $310(6.04)$ \\
\hline 4 & $3785(8.94)$ & $198 \mid(9.53)$ & 1159 (8.97) & $296(8.49)$ & $349(6.80)$ \\
\hline$\geq 5$ & $23,890(56.44)$ & $10,276(49.43)$ & $7716(59.70)$ & $2182(62.61)$ & $3716(72.39)$ \\
\hline Rate PPPY $(95 \% \mathrm{Cl})$ & $6.87(6.81,6.93)$ & $5.84(5.77,5.92)$ & $7.22(7.11,7.32)$ & $7.63(7.42,7.84)$ & $9.64(9.43,9.85)$ \\
\hline
\end{tabular}

Abbreviations: $\mathrm{Cl}$, confidence interval; COPD, chronic obstructive pulmonary disease; GOLD, Global Initiative for Chronic Obstructive Lung Disease; GP, general practitioner; PPPY, per patient per year.

analyses showed that the risk of a moderate or severe exacerbation in the year following the index date increased in line with COPD severity, such that $68.5 \%$ of patients in GOLD group D had a moderate COPD exacerbation compared with $27.3 \%$ in GOLD group A (HR: 3.84 ; $95 \%$ CI: $3.68,4.01$ ) (Table 3). Moderate exacerbations occurred in $62.3 \%$ of patients in GOLD group C (HR vs group A: 3.18; $95 \%$ CI: $3.03,3.34$ ) and $35.6 \%$ of those in GOLD group B (HR vs group A: $1.39 ; 95 \%$ CI: $1.34,1.44$ ). In addition, $17.8 \%$ of patients in GOLD group D had a severe COPD exacerbation compared with $2.6 \%$ in group A (HR: 7.32; 95\% CI: 6.58, 8.14); while severe exacerbations occurred in $6.5 \%$ of patients in group C (HR vs group A: 2.53 ; $95 \%$ CI: $2.16,2.95$ ) and $8.0 \%$ of those in group B (HR vs group A: $3.12 ; 95 \%$ CI: 2.81, 3.46).

The risk of death in the 12 months following the index date according to GOLD group was highest in GOLD groups D and B (the groups with the highest symptom burden at baseline), such that $7.8 \%$ of patients in group D died compared with $1.9 \%$ in group A (HR: $4.21 ; 95 \%$ CI: $3.67,4.84)$. Death occurred in $2.8 \%$ of patients in GOLD group C (HR vs group A: 1.46 ; $95 \%$ CI: 1.17 ,
1.83 ) and $5.4 \%$ of patients in group B (HR vs group A: 2.89 ; 95\% CI: $2.56,3.27$ ).

\section{Risk of COPD Exacerbations and Death in Patients with COPD According to MRC Dyspnea Grade}

Time to first moderate or severe COPD exacerbation, and time to death in patients stratified according to MRC dyspnea grade is shown in Figure $3 \mathrm{~A}-\mathrm{C}$. Univariate regression analyses showed that the risk of a moderate or severe exacerbation in the year following the index date increased in line with dyspnea severity, such that $52.0 \%$ of patients with MRC grade 5 dyspnea had a moderate COPD exacerbation compared with $25.3 \%$ of those with grade 1 dyspnea (HR: 2.71; 95\% CI: 2.46, 2.98) (Table 4). In addition, moderate exacerbations occurred in $48.5 \%$ of patients with grade 4 dyspnea (HR vs grade 1: $2.32 ; 95 \%$ CI: $2.19,2.46), 42.3 \%$ with grade 3 dyspnea (HR vs grade 1: $1.90 ; 95 \%$ CI: $1.80,2.00$ ), and $35.3 \%$ with grade 2 dyspnea (HR vs grade 1: $1.50 ; 95 \%$ CI: $1.42,1.58$ ). Of patients with grade 5 dyspnea, $25.3 \%$ had a severe COPD 

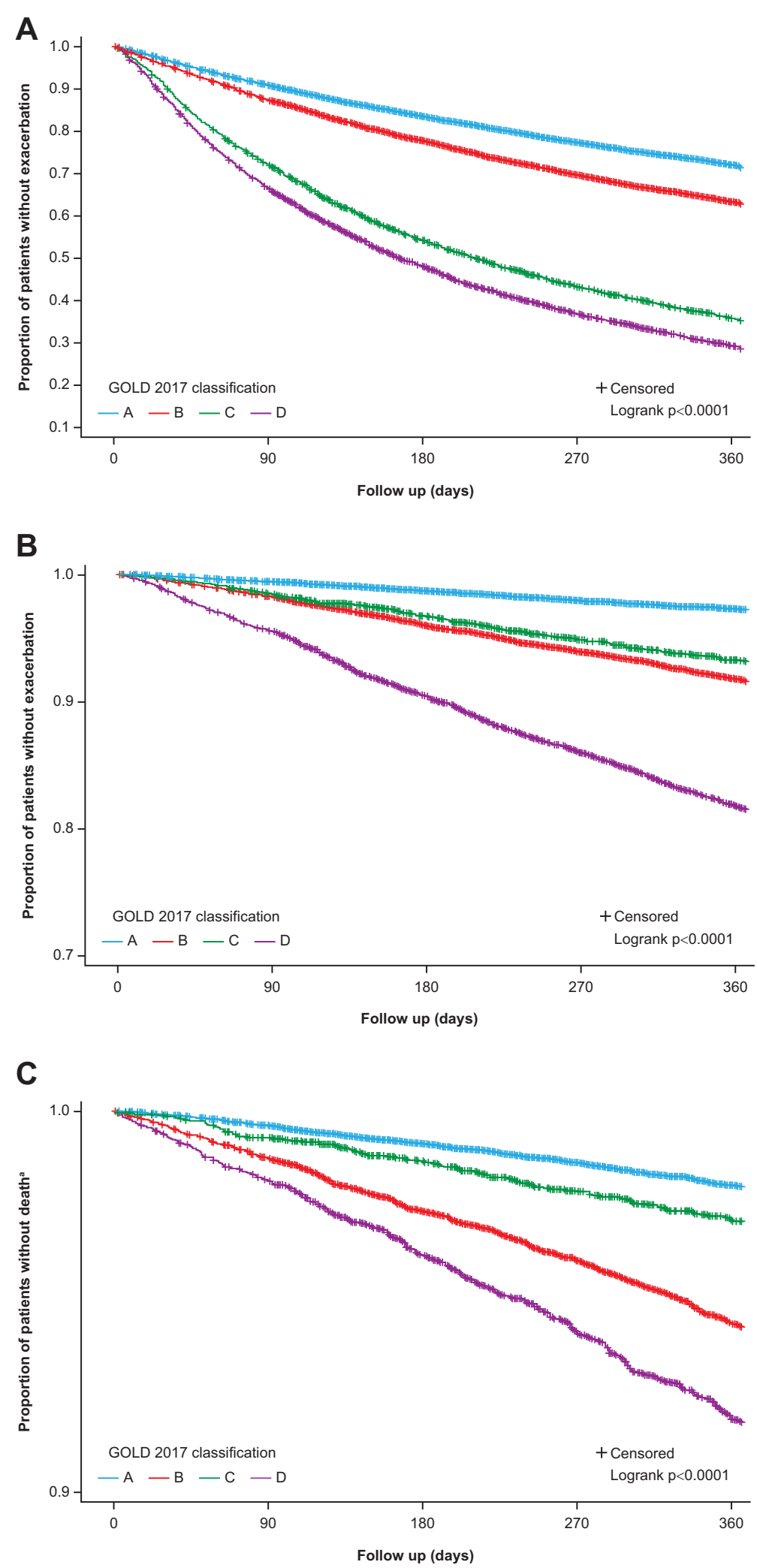

Figure 2 Univariate Cox proportional hazards model for time to the following events according to GOLD classification during the 12 months following the index date, (A) moderate COPD exacerbations; (B) severe COPD exacerbations; (C) death. ${ }^{2}$ Patients were followed up for death from 90 days post index date until 12 months post index date.

Note: $P$ values were generated from a single Log rank test comparing all groups.

Abbreviations: COPD, chronic obstructive pulmonary disease; GOLD, Global Initiative for Chronic Obstructive Lung Disease. 
Table 3 Time-to-Event Analysis for COPD Exacerbations and Death in the 12 Months After the Index Date According to GOLD 2017 Group

\begin{tabular}{|l|c|c|c|c|}
\hline & $\begin{array}{c}\text { GOLD Group } \\
\text { A (N=20,788) }\end{array}$ & $\begin{array}{c}\text { GOLD Group } \\
\text { B (N=12,925) }\end{array}$ & $\begin{array}{c}\text { GOLD Group } \\
\text { C (N=3485) }\end{array}$ & $\begin{array}{c}\text { GOLD Group } \\
\text { D (N=5 I33) }\end{array}$ \\
\hline $\begin{array}{l}\text { Moderate COPD exacerbation } \\
\text { No. with event (\%) } \\
\text { HR (95\% CI) }\end{array}$ & $5670(27.3)$ & $4597(35.6)$ & $2172(62.3)$ & $3517(68.5)$ \\
\hline $\begin{array}{l}\text { Severe COPD exacerbation } \\
\text { No. with event (\%) } \\
\text { HR (95\% CI) }\end{array}$ & 1 & $1.39(1.34,1.44)$ & $3.18(3.03,3.34)$ & $3.84(3.68,4.01)$ \\
\hline $\begin{array}{l}\text { Death } \\
\text { No. with event (\%) } \\
\text { HR (95\% CI) }\end{array}$ & $545(2.6)$ & $1033(8.0)$ & $228(6.5)$ & $913(17.8)$ \\
\hline
\end{tabular}

Notes: anadjusted Cox proportional HR calculated relative to GOLD Group A; 'Patients were followed up for death from 90 days post index date until 12 months post index date.

Abbreviations: $\mathrm{Cl}$, confidence interval; COPD, chronic obstructive pulmonary disease; GOLD, Global Initiative for Chronic Obstructive Lung Disease; HR, hazard ratio.

exacerbation versus $2.2 \%$ of those with grade 1 dyspnea (HR: 13.28 ; 95\% CI: 10.90, 16.17), while severe exacerbations occurred in $13.7 \%$ of patients with grade 4 dyspnea (HR vs grade 1: 6.66; 95\% CI: 5.63, 7.89), $7.8 \%$ with grade 3 dyspnea (HR vs grade 1: 3.65 ; 95\% CI: 3.08 , 4.32 ), and $3.6 \%$ with grade 2 dyspnea (HR vs grade 1 : 1.67; 95\% CI: $1.40,1.98)$.

The risk of death in the 12 months following the index date according to MRC dyspnea grade was highest in patients with the most severe symptoms of breathlessness, such that $15.7 \%$ of patients with grade 5 dyspnea died compared with $1.6 \%$ of those with grade 1 dyspnea (HR: 10.42 ; $95 \%$ CI: $8.23,13.19$ ), while death occurred in $7.7 \%$ of patients with grade 4 dyspnea (HR vs grade 1: 4.84; 95\% CI: $3.96,5.92$ ), $4.3 \%$ with grade 3 dyspnea (HR vs grade 1: $2.65 ; 95 \%$ CI: $2.17,3.24$ ), and $2.2 \%$ with grade 2 dyspnea (HR vs grade 1: 1.34; 95\% CI: 1.10, 1.65).

\section{Maintenance Therapy Use}

Maintenance therapy use during the 90 days on or after the index date in GOLD 2017 groups is presented in Figure 4. In this period, 9502 (22.4\%) patients (including 2043 [15.8\%] and 233 [4.5\%] in GOLD groups B and D, respectively) received no long-acting maintenance therapy. Overall, and across GOLD 2017 groups A-D, the most frequent prescribed maintenance therapies were ICS+LABA therapy and multiple-inhaler triple therapy, irrespective of GOLD classification. Overall, and across GOLD 2017 groups AD, LAMA only was the third most common prescribed treatment, while the least common prescribed treatments were ICS+LAMA and LAMA+LABA (Figure 4).

\section{Discussion}

This large observational study investigated COPD patients managed in primary care in England according to GOLD 2017 criteria. $^{5}$ A time-to-event analysis of patients categorized by GOLD 2017 criteria showed that patients in the highest-risk GOLD group D (indicating most severe disease) were almost four times more likely to have a moderate COPD exacerbation than those in GOLD group A during the year following the index date (HR: 3.84). The association between GOLD group $\mathrm{D}$ and risk of a severe exacerbation was particularly high: patients in GOLD group D were seven times more likely to experience a severe event leading to hospitalization compared with patients in group A (HR: 7.32). These results are consistent with other studies showing that exacerbation frequency predicts long-term exacerbation rates. ${ }^{10,12,13}$

The additional time-to-event analysis of COPD patients categorized according to MRC dyspnea grade suggests that breathlessness contributes to exacerbation severity and frequency. In our analysis, higher MRC dyspnea grades predicted an increased risk of exacerbations, especially severe exacerbations, over 12 months. Patients with MRC grade 5 dyspnea had a more than 2.5-fold higher risk (HR: 2.71) of moderate/severe exacerbation and a thirteenfold higher risk (HR: 13.28) of severe exacerbation compared with those with grade 1 dyspnea. Our results are in line with other studies that have shown an association between the severity of dyspnea and exacerbation severity and/or frequency, ${ }^{15,16,18,19}$ Our data showing an increasing HR for death in patients with dyspnea of increasing MRC 


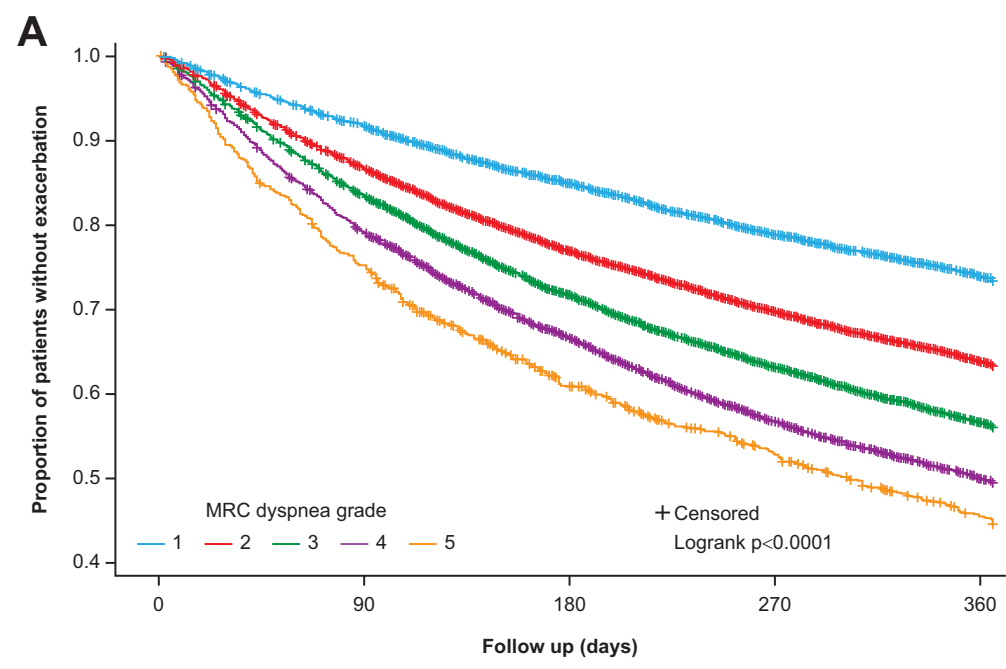

B

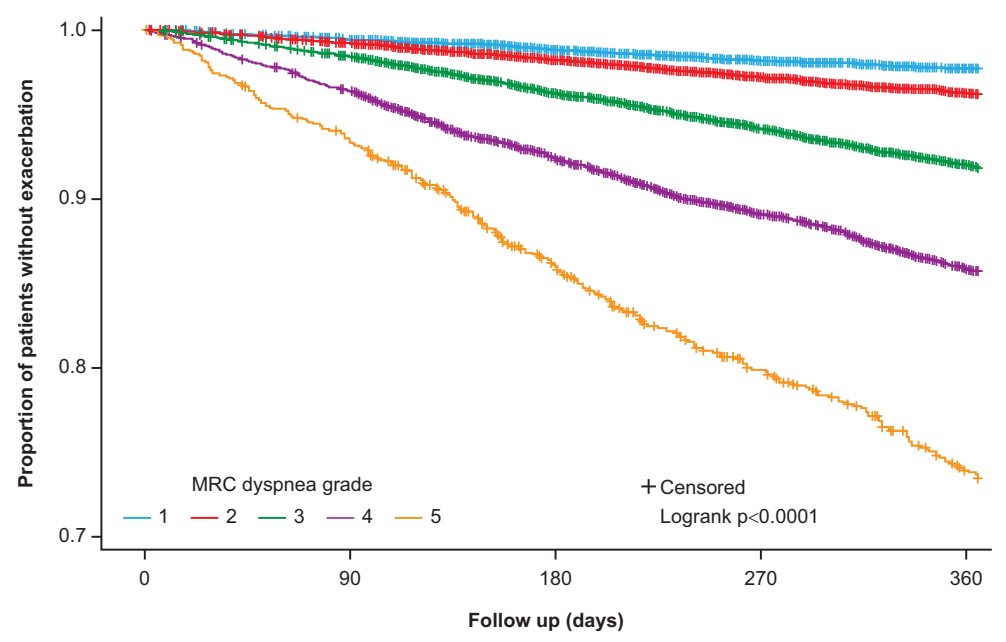

C

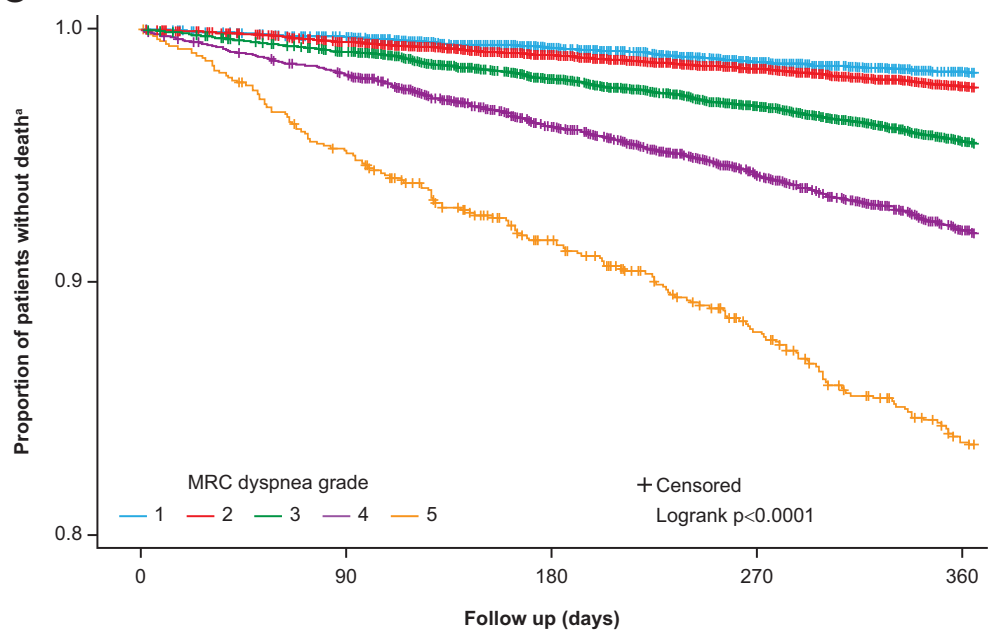

Figure 3 Univariate Cox proportional hazards model for time to the following events according to MRC dyspnea grade during the 12 months following the index date, (A)

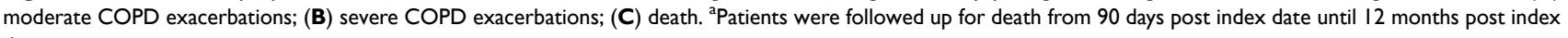
date.

Note: $P$ values were generated from a single Log rank test comparing all groups.

Abbreviations: COPD, chronic obstructive pulmonary disease; MRC, Medical Research Council. 
Table 4 Time-to-Event Analysis for COPD Exacerbations and Death in the 12 Months After the Index Date According to MRC Dyspnea Grade

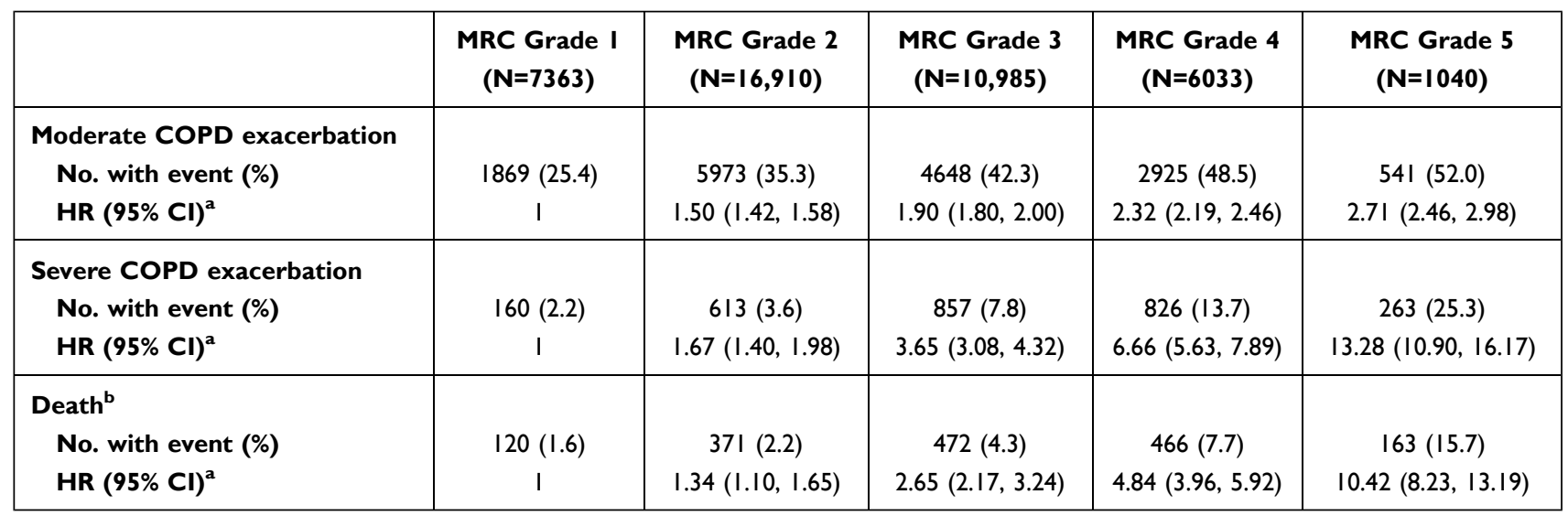

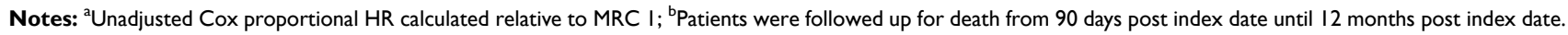
Abbreviations: $\mathrm{Cl}$, confidence interval; COPD, chronic obstructive pulmonary disease; HR, hazard ratio; MRC, Medical Research Council.

grade is consistent with findings from a recent study of patients in Sweden. ${ }^{25}$

In the time-to-event analysis by GOLD 2017 criteria, we found that GOLD group B patients had a higher risk of severe exacerbation than GOLD group $\mathrm{C}$ patients, which suggests that the current GOLD classification system may not adequately describe the risk of exacerbation for GOLD group $\mathrm{B}$ patients. Consistent with this, a previous study reported that the average number of severe exacerbations per year was higher among GOLD group B patients, as

70

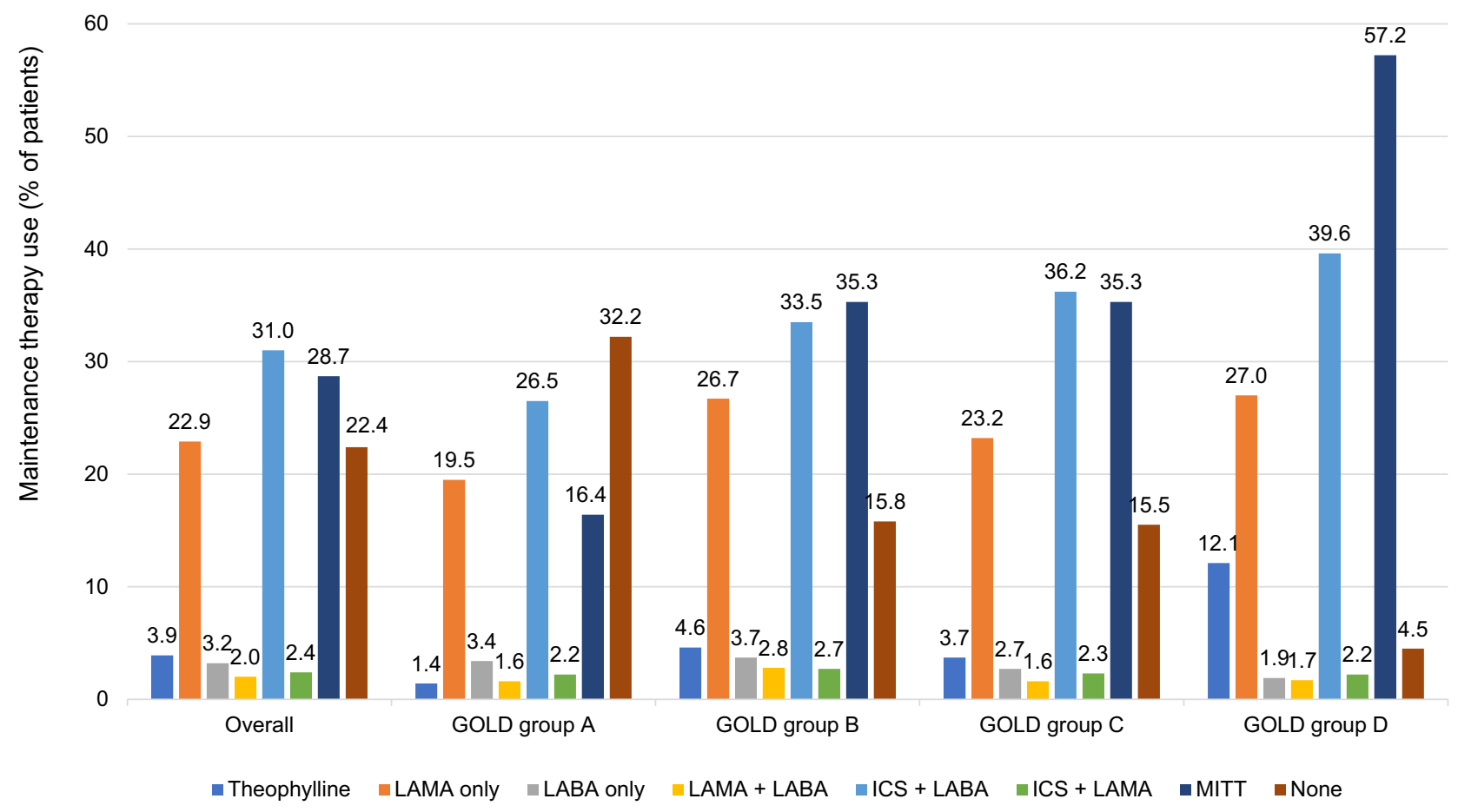

Figure 4 Maintenance therapy use, during the 90 days on or after the index date, overall and stratified by GOLD 2017 group.

Note: Patients may be included in more than one treatment category if they changed their maintenance therapy during the 90 -day period.

Abbreviations: GOLD, Global Initiative for Chronic Obstructive Lung Disease; ICS, inhaled corticosteroid; LABA, long-acting $\beta_{2}$-agonist; LAMA, long-acting muscarinic antagonist; MITT, multiple-inhaler triple therapy. 
compared with GOLD group C patients, although the odds ratio of severe exacerbation was higher among GOLD group $\mathrm{C}$ patients. ${ }^{26}$ Our finding that a higher MRC dyspnea score is associated with a graded increase in the risk of exacerbation suggests that breathlessness may be a useful tool to guide clinicians on the risk of exacerbations in GOLD group B patients. Longitudinal data in patients classified according to GOLD 2017 were consistent with the time-to-event analysis. Patients in GOLD group D experienced the largest burden of exacerbations and, out of all GOLD groups, were the most likely to visit their GP at least five times during the 12-month follow-up period.

Our analyses also investigated risk stratification by GOLD 2017 classification for mortality within 1 year of the index date. One-year mortality was highest in patients in GOLD groups D and B, the groups with the highest symptom burden at baseline. Patients in GOLD group $\mathrm{D}$ had a four times higher risk of death compared with GOLD group A patients (HR: 4.21), while those in GOLD group B had an almost three times higher risk of death at 1 year (HR: 2.89) compared with GOLD group A patients. Patients in GOLD group $C$ had 1.5 times the risk of death (HR: 1.46), when compared with GOLD group A patients. The higher mortality risk among GOLD group B patients than group $\mathrm{C}$ patients is consistent with findings from previous studies. ${ }^{26-28}$ In a cohort of $>6500$ Danish COPD patients stratified according to GOLD 2011 group, both 1 - and 3-year mortality rates were higher among GOLD group B patients than GOLD group C patients. ${ }^{27}$ Similarly, all-cause and respiratory mortality odds ratios were higher among GOLD 2011 group B patients compared with GOLD group $\mathrm{C}$ patients in the GenKOLS study. $^{28}$ All-cause and respiratory mortality rates were also found to be higher among GOLD 2017 group $\mathrm{B}$ patients than GOLD group $\mathrm{C}$ patients in a retrospective study in Taiwan. ${ }^{26}$ In addition, the 1 -year mortality rate of $8 \%$ we found in GOLD group D patients is broadly consistent with reports from similar studies, which range from $4.3 \%$ to $15.5 \%,{ }^{17,29}$ and our finding that the 1-year mortality risk is highest in GOLD groups $\mathrm{D}$ and $\mathrm{B}$ is consistent with a large Danish study that investigated the ability of GOLD 2017 classifications to predict all-cause and respiratory mortality. ${ }^{17}$ The Danish study also found that the risk of 3-year mortality compared with group A was higher for groups D and B than group C. ${ }^{17}$ In addition, its finding that 3-year mortality increased with increasing exacerbations and dyspnea is consistent with our findings that the risk of death at 1 year was associated with the severity of dyspnea, with mortality risk increasing in line with increasing MRC grade.

Approximately one-fifth $(22.4 \%)$ of the overall population had not received any long-acting maintenance therapy in the 12 months prior to the index date. During the 90 days on or after the index date, $22.4 \%$ of patients (including $15.8 \%$ and $4.5 \%$ in GOLD groups $\mathrm{B}$ and $\mathrm{D}$, respectively) did not receive any long-acting maintenance therapy. These results indicate that a significant minority of COPD patients remain untreated despite a considerable symptomatic burden. Further education and support may help healthcare practitioners to follow international and UK evidence-based recommendations on use of maintenance therapy for patients with COPD. ${ }^{24,30}$

The finding that patients in GOLD group B are at high risk of severe exacerbation warrants further investigation. Future research should focus on this group of patients and should aim to investigate what factors may be driving the risk of severe events beyond breathlessness, for example, worse airflow limitation or comorbidities. It has previously been suggested that underlying comorbidities, particularly cardiovascular disease, may contribute to the increased risk of mortality observed in GOLD group B patients. ${ }^{27}$ Further research may support the identification of a treatable trait in this group, which may help identify patients who are at higher risk of exacerbation, and may benefit from earlier, more intensive therapy.

A strength of this study is its inclusion of a large number of patients, comparable in magnitude to previous studies that have stratified patients by GOLD group, ${ }^{12,17,31}$ and its use of validated CPRD data which have, in previous studies, ${ }^{20,21}$ been used to confirm the completeness and high quality of computer-recorded, COPD-related GP data. Our study has been one of only a few to date that provide HR data for exacerbation or death in patients stratified by breathlessness. The use of $\mathrm{FEV}_{1}$ values also allowed COPD patients to be identified with greater sensitivity. It should be noted that pre- or post-bronchodilator status is not always captured in primary care data and, therefore, misclassification of disease is a possibility, however, a validated algorithm with a high positive predictive value was used in this study, to help mitigate this risk. In addition, determining factors associated with mortality risk was beyond the scope of the current study and unfortunately causes of death were 
not recorded. Identifying factors associated with mortality risk among COPD patients is an important topic that should be investigated in future studies. Other limitations of this study stem from its inclusion of patients from a single country, England, and differences in patient characteristics and clinical practice standards may apply in other countries. Moreover, regional differences in prescribing practices and prescription costs may lead to differences in baseline medication use, such that our data are not necessarily applicable on a broader scale.

\section{Conclusions}

Our study has demonstrated that there is clinical utility in stratifying this large primary care population according to GOLD criteria, with classification predicting the risk of moderate and severe COPD exacerbations. The risk of death within 1 year was most strongly predicted by the groups with the highest symptom burden at baseline (GOLD groups $\mathrm{B}$ and D). The observation that MRC dyspnea grade also predicted exacerbation severity and death suggests that breathlessness is a useful indicator of future outcomes and may be used to guide future risk management strategies, particularly in GOLD group $B$ patients who have a large symptom burden and who may benefit from early, intensive therapeutic intervention. It is hoped that an improved understanding of subpopulations of patients with COPD, and their respective burdens of disease and healthcare resource utilization in clinical practice, may enable the optimization of patient care.

\section{Abbreviations}

CI, confidence interval; COPD, chronic obstructive pulmonary disease; CPRD, Clinical Practice Research Datalink; $\mathrm{FEV}_{1}$, forced expiratory volume in 1 second; FVC, forced vital capacity; GOLD, Global Initiative for Chronic Obstructive Lung Disease; GP, general practitioner; HES, Hospital Episode Statistics; HR, hazard ratio; ICS, inhaled corticosteroid; ISAC, Independent Scientific Advisory Committee; LABA, long-acting $\beta_{2^{-}}$ agonist; LAMA, long-acting muscarinic antagonist; MITT, multiple-inhaler triple therapy; MRC, Medical Research Council; NHS, National Health Service; PPPY, per patient per year; SABA, short-acting $\beta_{2}$-agonist; SAMA, short-acting muscarinic antagonist; SD, standard deviation.

\section{Data Sharing Statement}

These data are not publicly available. To access similar data, interested researchers can apply to Clinical Practice Research Datalink (https://www.cprd.com/home) via the Independent Scientific Advisory Committee (ISAC).

\section{Ethics Approval and Informed Consent}

The study protocol was approved by the Independent Scientific Advisory Committee (ISAC), which reviewed the protocol and approved access to Clinical Practice Research Datalink data (ISAC study no. 17_270). Patient consent was not required as anonymized patient-level data were used in this analysis. Generic ethical approval for observational research using the CPRD with approval from ISAC has been granted by a Health Research Authority Research Ethics Committee (East MidlandsDerby, REC reference number 05/MRE04/87).

\section{Acknowledgments}

Editorial support (in the form of writing assistance, including preparation of the draft manuscript under the direction and guidance of the authors, collating and incorporating authors' comments for each draft, assembling tables and figures, grammatical editing and referencing) was provided by Joanna Wilson, $\mathrm{PhD}$, of Gardiner Caldwell Communications (Macclesfield, UK) and Caroline McGown, PhD, of Aura, a division of Spirit Medical Communications Group Limited, and was funded by GlaxoSmithKline. Data from this study have previously been presented in abstract/poster form at the British Thoracic Society Winter Meeting, London, UK, Dec 58, 2018 (Sansbury LB, Anley G, Bains C, Ho S, Ismaila AS. P68 Disease burden, treatment, and health care utilisation of COPD patients by their GOLD classification in the UK. Thorax 2018;73:A138-A139). This study is based, in part, on data from the Clinical Practice Research Datalink obtained under license from the UK Medicines and Healthcare products Regulatory Agency. The data is provided by patients and collected by the NHS as part of their care and support. The interpretation and conclusions contained in this study are those of the authors alone. Copyright (C) (2020), re-used with the permission of The Health \& Social Care Information Centre. All rights reserved.

\section{Author Contributions}

All authors made a significant contribution to the work reported, whether that was in the conception, study design, 
execution, acquisition of data, analysis and interpretation, or in all these areas; took part in drafting, revising or critically reviewing the manuscript; gave final approval of the version to be published; have agreed on the journal to which the article has been submitted; and agree to be accountable for all aspects of the work.

\section{Funding}

This study was funded by GlaxoSmithKline (study number 208979). The sponsor was involved in study conception and design, data interpretation, and the decision to submit the article for publication. The sponsor was also given the opportunity to review the manuscript for medical and scientific accuracy as well as intellectual property considerations.

\section{Disclosure}

The authors declare the following conflicts of interest during the last three years in relation to this article: KJR, CC, GA, and ASI are employees of, and hold shares in, GlaxoSmithKline. LBS was an employee of, and held shares in, GlaxoSmithKline, during the time the study was conducted. GA and KJR are currently affiliated with Speciality \& Primary Care, GlaxoSmithKline, Brentford, UK. LBS is currently affiliated with Medical Affairs, Ultragenyx Pharmaceutical Inc., Novato, CA, USA. ASI is also an unpaid part-time professor at McMaster University, Hamilton, ON, Canada. CB was an employee of GlaxoSmithKline during the time the study was conducted. The authors report no other conflicts of interest in this work.

\section{References}

1. Rabe KF, Watz H. Chronic obstructive pulmonary disease. Lancet. 2017;389(10082):1931-1940. doi:10.1016/S0140-6736(17)31222-9

2. Chapman KR, Mannino DM, Soriano JB, et al. Epidemiology and costs of chronic obstructive pulmonary disease. Eur Respir J. 2006;27(1):188-207. doi:10.1183/09031936.06.00024505

3. World Health Organization. The top 10 causes of death; 2018. Available from: https://www.who.int/news-room/fact-sheets/detail/thetop-10-causes-of-death. Accessed November 4, 2020.

4. Vestbo J, Hurd SS, Agustí AG, et al. Global strategy for the diagnosis, management, and prevention of chronic obstructive pulmonary disease: GOLD executive summary. Am J Respir Crit Care Med. 2013;187(4):347-365. doi:10.1164/rccm.201204-0596PP

5. Vogelmeier CF, Criner GJ, Martinez FJ, et al. Global strategy for the diagnosis, management, and prevention of chronic obstructive lung disease 2017 report: GOLD executive summary.. Eur Respir J. 2017;49(3):1700214. doi:10.1183/13993003.00214-2017

6. Cabrera López C, Casanova Macario C, Marín Trigo JM, et al. Comparison of the 2017 and 2015 Global Initiative for Chronic Obstructive Lung Disease reports. Impact on grouping and outcomes. Am J Respir Crit Care Med. 2018;197(4):463-469. doi:10.1164/ rccm.201707-1363OC
7. Criner RN, Labaki WW, Regan EA, et al. Mortality and exacerbations by Global Initiative for Chronic Obstructive Lung Disease groups ABCD: 2011 versus 2017 in the COPDGene ${ }^{\circledR}$ cohort. Chronic Obstr Pulm Dis. 2019;6(1):64-73.

8. Högman M, Sulku J, Ställberg B, et al. 2017 Global Initiative for Chronic Obstructive Lung Disease reclassifies half of COPD subjects to lower risk group. Int $J$ Chron Obstruct Pulmon Dis. 2018;13:165-173. doi:10.2147/COPD.S151016

9. Halpin DMG, Decramer M, Celli B, Kesten S, Liu D, Tashkin DP. Exacerbation frequency and course of COPD. Int J Chron Obstruct Pulmon Dis. 2012;7:653-661. doi:10.2147/COPD.S34186

10. Hurst JR, Vestbo J, Anzueto A, et al. Susceptibility to exacerbation in chronic obstructive pulmonary disease. $N$ Engl J Med. 2010;363 (12):1128-1138. doi:10.1056/NEJMoa0909883

11. Müllerova H, Maselli DJ, Locantore N, et al. Hospitalized exacerbations of COPD: risk factors and outcomes in the ECLIPSE cohort. Chest. 2015;147(4):999-1007. doi:10.1378/ chest.14-0655

12. Müllerová H, Shukla A, Hawkins A, Quint J. Risk factors for acute exacerbations of COPD in a primary care population: a retrospective observational cohort study. BMJ Open. 2014;4(12):e006171. doi:10.1136/bmjopen-2014-006171

13. Rothnie KJ, Müllerová H, Smeeth L, Quint JK. Natural history of chronic obstructive pulmonary disease exacerbations in a general practice-based population with chronic obstructive pulmonary disease. Am J Respir Crit Care Med. 2018;198(4):464-471. doi:10.1164/rccm.201710-2029OC

14. Schmidt SAJ, Johansen MB, Olsen M, et al. The impact of exacerbation frequency on mortality following acute exacerbations of COPD: a registry-based cohort study. BMJ Open. 2014;4(12): e006720. doi:10.1136/bmjopen-2014-006720

15. Agusti A, Edwards LD, Celli B, et al. Characteristics, stability and outcomes of the 2011 GOLD COPD groups in the ECLIPSE cohort. Eur Respir J. 2013;42(3):636-646. doi:10.1183/090319 36.00195212

16. Calverley PM, Tetzlaff K, Dusser D, et al. Determinants of exacerbation risk in patients with COPD in the TIOSPIR study. Int $J$ Chron Obstruct Pulmon Dis. 2017;12:3391-3405. doi:10.2147/COPD. S145814

17. Gedebjerg A, Szépligeti SK, Wackerhausen LH, et al. Prediction of mortality in patients with chronic obstructive pulmonary disease with the new Global Initiative for Chronic Obstructive Lung Disease 2017 classification: a cohort study. Lancet Respir Med. 2018;6(3):204-212. doi:10.1016/S2213-2600(18)30002-X

18. Punekar YS, Mullerova H, Small M, et al. Prevalence and burden of dyspnoea among patients with chronic obstructive pulmonary disease in five European countries. Pulm Ther. 2016;2(1):59-72. doi:10.1007/s41030-016-0011-5

19. Sansbury LB, Anley G, Bains C, Ho S, Ismaila A. Disease burden, treatment, and health care utilisation of COPD patients by their GOLD classification in the U.K. Thorax. 2018;73(Supp14):A138A139. [Abstract P68].

20. Quint JK, Mullerova H, DiSantostefano RL, et al. Validation of chronic obstructive pulmonary disease recording in the Clinical Practice Research Datalink (CPRD-GOLD). BMJ Open. 2014;4(7): e005540. doi:10.1136/bmjopen-2014-005540

21. Rebordosa C, Plana E, Aguado J, et al. GOLD assessment of COPD severity in the Clinical Practice Research Datalink (CPRD). Pharmacoepidemiol Drug Saf. 2019;28(2):126-133. doi:10.1002/ pds. 4448

22. Chalmers JD, Poole C, Webster S, Tebboth A, Dickinson S, Gayle A. Assessing the healthcare resource use associated with inappropriate prescribing of inhaled corticosteroids for people with chronic obstructive pulmonary disease (COPD) in GOLD groups A or B: an observational study using the Clinical Practice Research Datalink (CPRD). Respir Res. 2018;19(1):63. 
23. Rothnie KJ, Mullerova H, Thomas SL, et al. Recording of hospitalizations for acute exacerbations of COPD in UK electronic health care records. Clin Epidemiol. 2016;8:771-782. doi:10.2147/CLEP.S117867

24. National Institute for Health and Clinical Care Excellence. Chronic obstructive pulmonary disease in over 16s: diagnosis and management; 2018. Available from: https://www.nice.org.uk/gui dance/ng115/resources/chronic-obstructive-pulmonary-disease-inover-16s-diagnosis-and-management-pdf-66141600098245. Accessed January 14, 2021.

25. Sandberg J, Engström G, Ekström M. Breathlessness and incidence of COPD, cardiac events and all-cause mortality: a 44-year follow-up from middle age throughout life. PLoS One. 2019;14(3):e0214083. doi:10.1371/journal.pone.0214083

26. Han MZ, Hsiue TR, Tsai SH, Huang TH, Liao XM, Chen CZ. Validation of the GOLD 2017 and new 16 subgroups (1A-4D) classifications in predicting exacerbation and mortality in COPD patients. Int J Chron Obstruct Pulmon Dis. 2018;13:3425-3433. doi:10.2147/COPD.S179048

27. Lange P, Marott JL, Vestbo J, et al. Prediction of the clinical course of chronic obstructive pulmonary disease, using the new GOLD classification: a study of the general population. Am J Respir Crit Care Med. 2012;186(10):975-981. doi:10.1164/rccm.201207-1299OC
28. Johannessen A, Nilsen RM, Storebø M, Gulsvik A, Eagan T, Bakke P. Comparison of 2011 and 2007 Global Initiative for Chronic Obstructive Lung Disease guidelines for predicting mortality and hospitalization. Am J Respir Crit Care Med. 2013;188 (1):51-59. doi:10.1164/rccm.201212-2276OC

29. Kobayashi S, Hanagama M, Ishida M, et al. Clinical characteristics and outcomes in Japanese patients with COPD according to the 2017 GOLD classification: the Ishinomaki COPD Network Registry. Int J Chron Obstruct Pulmon Dis. 2018;13:3947-3955. doi:10.21 47/COPD.S182905

30. Global Initiative for Chronic Obstructive Lung Disease. 2021 Global strategy for prevention, diagnosis and management of COPD; 2021. Available from: https:/goldcopd.org/2021-gold-reports/. Accessed January 14, 2021.

31. Soriano JB, Lamprecht B, Ramírez AS, et al. Mortality prediction in chronic obstructive pulmonary disease comparing the GOLD 2007 and 2011 staging systems: a pooled analysis of individual patient data. Lancet Respir Med. 2015;3(6):443-450. doi:10.1016/S22132600(15)00157-5

\section{Publish your work in this journal}

The International Journal of COPD is an international, peer-reviewed journal of therapeutics and pharmacology focusing on concise rapid reporting of clinical studies and reviews in COPD. Special focus is given to the pathophysiological processes underlying the disease, intervention programs, patient focused education, and self management protocols. This journal is indexed on PubMed Central, MedLine and CAS. The manuscript management system is completely online and includes a very quick and fair peer-review system, which is all easy to use. Visit http://www.dovepress.com/testimonials.php to read real quotes from published authors. 